Vietnam Journal of Mechanics, VAST, Vol.33, No. 3 (2011), pp. $162-169$

\title{
A FORMULA OF EVALUATING STRUCTURAL SAFETY BASED ON FUZZY SET THEORY
}

\author{
Le Xuan Huynh, Le Cong Duy \\ National University of Civil Engineering, Vietnam
}

\begin{abstract}
This article presents an approach to assess safety levels of structures. A new formula for determining the fuzzy reliability of structures is proposed for the case where the set of loading effect and set of structural durability are general fuzzy sets. Illustration example concerning the bending strength evaluation of a simple-beam structure, is presented with the choice of triangular fuzzy sets for loading effect and structural durability.

Key words: Fuzzy logic and application, fuzzy reliability.
\end{abstract}

\section{INTRODUCTION}

Data and models encountered in natural sciences and engineering are more or less characterized by uncertainty. The uncertainty models can be investigated by the fuzzy theory. Fuzzy theory has been known since 1965. Professor Lofti A. Zadeh had the first article presenting the fuzzy sets and fuzzy logic. It is firstly applied in electronic engineering, next in fields of computer sciences and control techniques. And since 1970, fuzzy set theory has been applied and developed to the fields of civil engineering and computational mechanics. In order to assess the safety of construction structures based on fuzzy models, now, there are two trends for approaching. The first trend, based on fuzzy probability theory is to establish methods to define the fuzzy reliability of structures [1]. Using the second trend, researchers propose different formulas to calculate the fuzzy reliability based on fuzzy set theory and random interference model [2-7]. This article presents a new formula to define the fuzzy reliability following the second trend. Numerical examples are compared with some formulas for estimating the safety of structures.

In [2], we present a formula to evaluate safety levels and/or failure levels of structures, in cases where loading effect and structural durability are two triangular fuzzy sets. Based on the mathematics of fuzzy logic, the failure ratio (FR) of structures is defined by the formulation: $\mathrm{FR}=\left(\omega_{R}+\omega_{S}\right) /\left(\Omega_{S}+\Omega_{R}\right)$, and the safety ratio (SR) of structures can be inferred: $\mathrm{SR}=1-\mathrm{FR}$, where $\Omega_{R}, \Omega_{S}$ is in turn full area of $\tilde{R}, \tilde{S}$; and $\left(\omega_{R}+\omega_{S}\right)$ is the area of intersection part of $\tilde{R}$ and $\tilde{S}$. A problem of the formula in [2] is finding the $\left(\omega_{R}+\omega_{S}\right)$, however in some cases it is very difficult.

N.D. Xan [4] uses fuzzy-random interferential model. Set of response of structure $\tilde{S}$ is described as a triangular fuzzy set with membership function $\mu(x)$, and ability of structure $\mathrm{R}$ is described as a random variable with standard distributed density function $f(x)$. Fuzzy 
incredibility $\widetilde{P}_{f}$ is defined by formula: $\widetilde{P}_{f}=\int \mu(x) \cdot f(x) d x$, and fuzzy reliability $\widetilde{P}_{S}$ can be

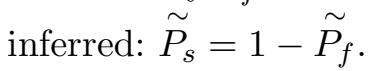

Changing characteristic of S and R, Kwan Ling Lai [5] uses random-fuzzy interferential model. Set of response of structure $\mathrm{S}$ is described as a random variable with standard distributed density function $f(x)$, and Ability of structure $R$ is described as a triangular Fuzzy set $\tilde{R}$ with membership function $\mu(x)$. Fuzzy incredibility $\widetilde{P}_{f}$ is defined by formulation: $\widetilde{P}_{f}=\int f(x) \cdot \mu(x) d x$, and Fuzzy reliability $\widetilde{P}_{S}$ can be inferred: $\widetilde{P}_{s}=1-\widetilde{P}_{f}$. Formulas in [4] and [5] are the approximate formulas, one member in the integral is a fuzzy set, other member is a random variable. Functions $\mu(x)$ and $f(x)$ are not of the same measurement, so these formulas [4], [5] give approach results. In the follow example, these formulas will not be used.

N.V. Pho [6] uses interferential model which is similar to random model. Set of response of structure $\tilde{S}$ and set of ability of structure $\tilde{R}$ are described as fuzzy sets with membership functions are triangular models. The formula consider the difference set $\tilde{M}=$ $\tilde{R}-\tilde{S}$ with membership function $\mu(m)$, then the author convert area of graphs set of $\mu(m)$ into new membership, area of which is equal to unit. The fuzzy incredibility $\widetilde{P}_{f}$ is calculated by the left-part area of the vertical axis of graphs of new membership function, and fuzzy

reliability $\widetilde{P}_{S}$ is the right-part area of the vertical axis of graphs of new membership function this mean $\widetilde{P}_{s}=1-\widetilde{\widetilde{P}}_{f}$.

Weimin Dong et all [7] directly uses set $\tilde{S}$ and set $\tilde{R}$ with corresponding triangular membership functions $\mu(s)$ and $\mu(r)$. Fuzzy failure possibility (FP) is calculated as formulation: $\mathrm{FP}=h / 2$, and fuzzy safety possibility (SP) is calculated as: $\mathrm{SP}=1-h / 2$, where $\mathrm{h}$ is the ordinate of intersectional point between two curves $\mu(s)$ and $\mu(r)$. Formula in [7] shows the way of calculating approximately, it only considers the height $h$ of the intersectional part area but hasn't calculated its width base $c$.

Based on fuzzy interferential model, in this article, authors propose a new formulation of safety assessment for structures, named: "Formula of area ratio".

\section{FORMULATION OF AREA RATIO}

The formulation for calculating fuzzy reliability of structures is established based on the idea of fuzzy interferential model, comparing the set of loading effect $\tilde{S}_{i}$ with the set of structural durability $\tilde{R}_{i}$. Consider set $\tilde{S}_{i}$ and set $\tilde{R}_{i}$ as fuzzy sets, in the real numbers field, with corresponding membership function $\mu_{\tilde{S}_{i}}(x)$ and $\mu_{\tilde{R}_{i}}(x)$ which in the general forms (Fig. 1).

For safety evaluate, comparing the set $\tilde{S}_{i}$ with the set $\tilde{R}_{i}$. We consider the difference set $\tilde{M}_{i}=\tilde{R}_{i}-\tilde{S}_{i}$. By the fuzzy interval analysis algorithm or $\alpha$ - level optimization of the extension principle [1], we define the membership functions $\mu_{\tilde{M}_{i}}(x)$ of fuzzy set $\tilde{M}_{i}$, can be as follow ( Fig. 2).

In the Fig. $2 \mathrm{a}$, we see that the membership function $\mu_{\tilde{M}_{i}}(x)$ is fully on the left of the vertical axis, this mean set of loading effect $\tilde{S}_{i}>$ set of structural durability $\tilde{R}_{i}$, member 

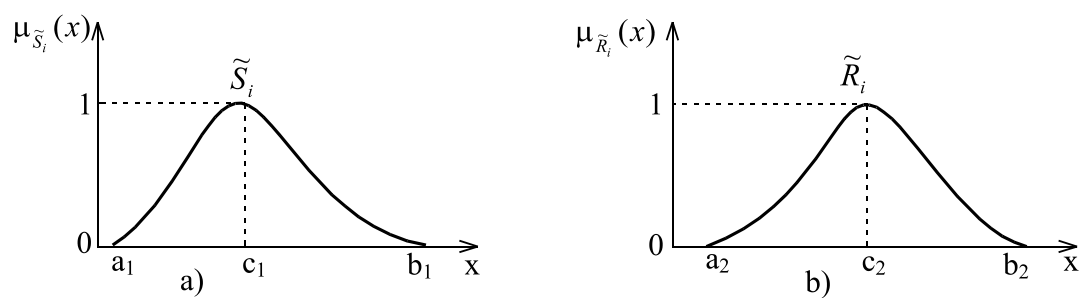

Fig. 1. Membership functions of set $\mathrm{Si}$ a) and of set Ri b)

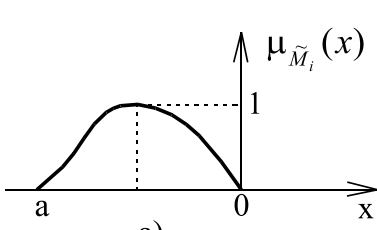

a)

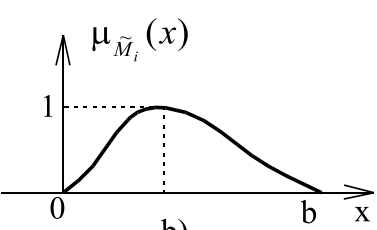

b)

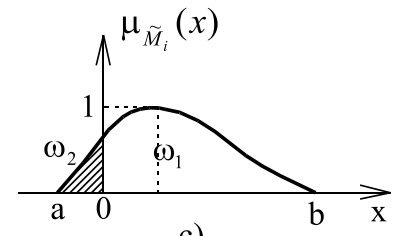

c)

Fig. 2. Cases of the set $\tilde{M}_{i}$

of structure is entirely failure or we say the failure ratio (FR) of the structural member is $100 \%$. On the contrary, in the Fig. $2 \mathrm{~b}$ we see the membership function $\mu_{\tilde{M}_{i}}(x)$ is fully on the right of the vertical axis, this mean set of loading effect $\tilde{S}_{i}<$ set of structural durability $\tilde{R}_{i}$, member of structure is entirely safety or we say the safety ratio (SR) of the structural member is $100 \%$

Generally, in the Fig. 2c, we see that the membership function $\mu_{\tilde{M}_{i}}(x)$ of fuzzy set $\tilde{M}_{i}$ has a part on the left and another part on the right of the vertical axis. This mean state of the structural member $\tilde{S}_{i}$ is not entirely safety, or we say it has a failure part, corresponding to the left-area from the vertical axis, and the safety part correspond to the right-area from the vertical axis.

So the reliability $\left(P_{s}^{i}\right)$ of the i-th structural member can be defined by a formulation proposed as follows:

$$
P_{s}^{i}=\frac{\omega_{1}}{\Omega_{M}}=\frac{\int_{0}^{b} \mu_{\tilde{M}_{i}}(x) d x}{\int_{a}^{b} \mu_{\tilde{M}_{i}}(x) d x}
$$

and the incredibility $\left(P_{f}^{i}\right)$ of structural member :

$$
P_{f}^{i}=\frac{\omega_{2}}{\Omega_{M}}=\frac{\int_{a}^{0} \mu_{\tilde{M}_{i}}(x) d x}{\int_{a}^{b} \mu_{\tilde{M} i}(x) d x}=1-P_{s}^{i}
$$

where $\omega_{1}$ is the right-area, $\omega_{2}$ is the left-area from the vertical axis, and $\Omega_{M}=\left(\omega_{1}+\omega_{2}\right)$ is the full area of graph $\mu_{\tilde{M}_{i}}(x)$. 
We see that $P_{s}^{i}+\mathrm{P}_{f}^{i}=1$.

After determining the reliability $P_{s}^{i}$ of $\mathrm{i}^{\text {th }}$ member of structural system, we can define the reliability of structural system by electric net schema, or follow reliability interval:

$$
\prod_{i=1}^{n} P_{s}^{i} \leq P_{s} \leq \min \left(P_{s}^{1}, P_{s}^{2}, \ldots, P_{s}^{n}\right)=P_{s}^{i} \min .
$$

\section{EXAMPLE OF APPLICATION}

Consider a simple example in order to test and to illustrate the proposed formula. In this example, the membership functions of $\mathrm{S}$ and $\mathrm{R}$ are triangular types, the most commonly used in engineering practice. A reinforced-concrete beam is shown in Fig. 3, where As $=3 \phi 18=7,63 \mathrm{~cm}^{2}$. Loads are triangular-fuzzy numbers ( Fig. 4). The problem is to evaluate strength safety level of the beam.

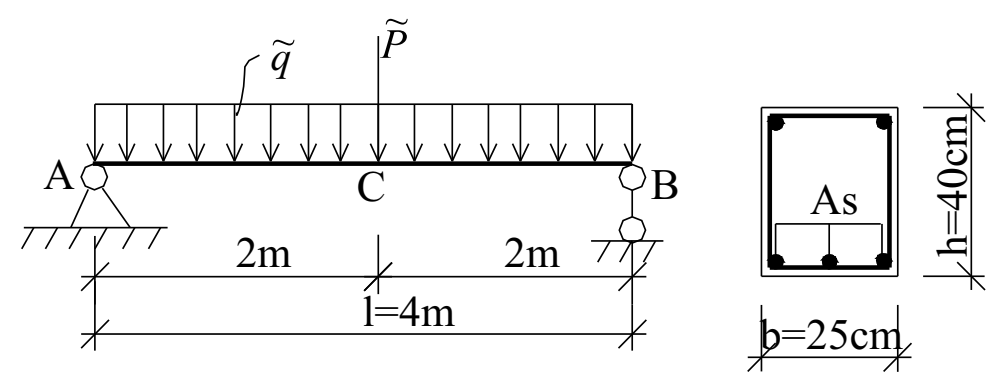

Fig. 3. Reinforced-concrete beam; loads, cross section
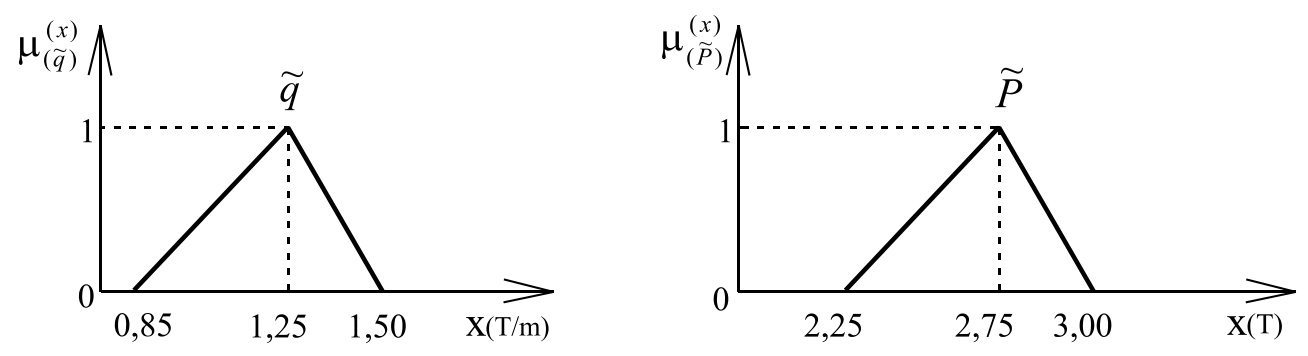

Fig. 4. Fuzzy loads membership functions

\subsection{Determination of membership functions of fuzzy moment at $\mathbf{C}$}

Based on structural methods, we apply the principle of load-contribution to define value of fuzzy moment at $\mathrm{C}$ as in Fig. 5 :

$$
\tilde{M}_{C}=\frac{l}{4} \tilde{P}+\frac{l^{2}}{8} \tilde{q}
$$




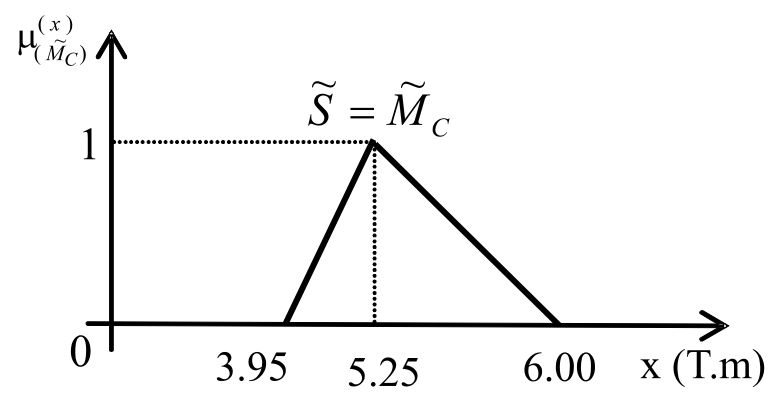

Fig. 5. Membership function of fuzzy moment at $C$

Application of fuzzy interval analysis to determine the membership functions of fuzzy moment at $C$.

\subsection{Construction of membership functions of fuzzy moment of designing section}

Variable matrix and moment matrix of section.

Designing section parameters: $b=25 \mathrm{~cm}, h=40 \mathrm{~cm}$, and area of steel rods $A_{s}=$ $3 \phi 18=7.63 \mathrm{~cm}^{2}$, using method of fuzzy linear regression to define membership function of moment of designing section $[\tilde{M}]$. Using method of fuzzy linear regression, we need to construct a variable matrix by changing values of random parameters with possible amplitude is $\pm 5 \%$ of mean value.

Changing values of random parameters, we have a matrix as follows (see Table 1):

Fuzzy linear regression function.

Equation of fuzzy linear regression function [9] is expressed:

$$
\begin{gathered}
y=[\tilde{M}]=\gamma_{1} x_{1}+\gamma_{2} x_{2}+\gamma_{3} x_{3}+\gamma_{4} x_{4}+\gamma_{5} x_{5}+\gamma_{6} x_{6} \\
y=[\tilde{M}]=\gamma_{1} \cdot b+\gamma_{2} \cdot h+\gamma_{3} \cdot A_{S}+\gamma_{4} \cdot b^{2}+\gamma_{5} \cdot h^{2}+\gamma_{6} \cdot A_{S}^{2}
\end{gathered}
$$

in which $\gamma_{j}\left(a_{j}, c_{j}\right)$ is a fuzzy component, we replace values of fuzzy component into equation (11) to have value of $y=[\tilde{M}]$, show the membership function of $[\tilde{M}]$. Based on Fuzzy Lest-Squares Linear Regression [9] to define the value of $a_{j}$ :

$$
a=\left[x^{T} \cdot x\right]^{-1}\left[x^{T} \cdot y\right]
$$

where $x$ is a variable matrix $(n \times k)=(27 \times 6)$ and $y$ is a moment matrix $(n \times 1)=(27 \times 1)$. Matrix $[c]$ is defined from restrain condition: total of errors of $c_{j}$ is minimized. So, we have a linear-optimal problem:

$$
Z=\sum_{i=1}^{n} c^{T}\left|x_{i}\right|^{T} \rightarrow \min
$$

subjected to:

$$
\left\{\begin{array}{l}
a^{T} x_{i}^{T}-(1-H) c^{T}\left|x_{i}\right|^{T} \leq y_{i} \\
a^{T} x_{i}^{T}+(1-H) c^{T}\left|x_{i}\right|^{T} \geq y_{i} \\
c_{j} \geq 0, \quad j=\overline{1, k}
\end{array}\right.
$$


Table 1. Values of random parameters

\begin{tabular}{|c|c|c|c|c|c|c|}
\hline \multicolumn{6}{|c|}{ Variable matrix } & Moment matrix $(y)$ \\
\hline$b(\mathrm{~cm})$ & $h(\mathrm{~cm})$ & $\mathrm{As}(\mathrm{cm})^{2}$ & $b^{2}(\mathrm{~cm})^{2}$ & $h^{2}(\mathrm{~cm})^{2}$ & $\mathrm{~A}_{S}^{2}(\mathrm{~cm})^{4}$ & $\mathrm{M}(\mathrm{kG} . \mathrm{m})$ \\
\hline 23.75 & 38 & 7.2485 & 564.0625 & 1444 & 52.54075 & 5415.252 \\
\hline 23.75 & 38 & 7.63 & 564.0625 & 1444 & 58.2169 & 5630.546 \\
\hline 23.75 & 38 & 8.0115 & 564.0625 & 1444 & 64.18413 & 5838.867 \\
\hline 23.75 & 40 & 7.2485 & 564.0625 & 1600 & 52.54075 & 5799.475 \\
\hline 23.75 & 40 & 7.63 & 564.0625 & 1600 & 58.2169 & 6034.592 \\
\hline 23.75 & 40 & 8.0115 & 564.0625 & 1600 & 64.18413 & 6262.697 \\
\hline 23.75 & 42 & 7.2485 & 564.0625 & 1764 & 52.54075 & 6184.383 \\
\hline 23.75 & $\overline{42}$ & 7.63 & 564.0625 & 1764 & 58.2169 & 6439.397 \\
\hline 23.75 & $\overline{42}$ & 8.0115 & 564.0625 & 1764 & 64.18413 & 6687.364 \\
\hline 25 & 38 & 7.2485 & 625 & 1444 & 52.54075 & 5478.174 \\
\hline 25 & 38 & $\begin{array}{l}7.63 \\
\end{array}$ & 625 & 1444 & 58.2169 & 5700.265 \\
\hline 25 & 38 & 8.0115 & 625 & 1444 & 64.18413 & 5915.733 \\
\hline 25 & 40 & 7.2485 & 625 & 1600 & 52.54075 & 5862.757 \\
\hline 25 & 40 & 7.63 & 625 & 1600 & 58.2169 & 6104.71 \\
\hline 25 & 40 & 8.0115 & 625 & 1600 & 64.18413 & 6340.003 \\
\hline 25 & 42 & 7.2485 & 625 & 1764 & 52.54075 & 6247.99 \\
\hline 25 & 42 & 7.63 & 625 & 1764 & 58.2169 & 6509.877 \\
\hline 25 & 42 & 8.0115 & 625 & 1764 & 64.18413 & 6765.067 \\
\hline 26.25 & 38 & 7.2485 & 689.0625 & 1444 & 52.54075 & 5535.104 \\
\hline 26.25 & 38 & 7.63 & 689.0625 & 1444 & $\begin{array}{l}58.2169 \\
\end{array}$ & 5763.345 \\
\hline 26.25 & 38 & 8.0115 & 689.0625 & 1444 & 64.18413 & 5985.279 \\
\hline 26.25 & 40 & 7.2485 & 689.0625 & 1600 & 52.54075 & 5920.012 \\
\hline 26.25 & 40 & 7.63 & 689.0625 & 1600 & 58.2169 & 6168.151 \\
\hline 26.25 & 40 & 8.0115 & 689.0625 & 1600 & 64.18413 & 6409.946 \\
\hline 26.25 & 42 & 7.2485 & 689.0625 & 1764 & 52.54075 & 6305.54 \\
\hline 26.25 & $\overline{42}$ & 7.63 & 689.0625 & 1764 & 58.2169 & 6573.643 \\
\hline 26.25 & 42 & 8.0115 & 689.0625 & 1764 & 64.18413 & 6835.37 \\
\hline
\end{tabular}

Where $\left.c=\left[\begin{array}{lllll}c_{1} & c_{2} & c_{j} & \ldots & c_{k}\end{array}\right]^{T}\right], y_{i}$ is a moment vector (row $i$ ) of matrix $y, x_{i}$ is a variable matrix (row $i$ ) of matrix $x$, and $H$ is fuzzy threshold which has value $\in[0,1]$.

We assume $H=0.5$ and change $(7),(8)$ into:

$$
Z=\sum_{j=1}^{6} c_{j}\left(\sum_{i=1}^{27} x_{i j}\right) \rightarrow \min
$$

subjected to:

$$
\left\{\begin{array}{l}
-c^{T}\left|x_{i}\right|^{T} \leq\left(y_{i}-a^{T} x_{i}^{T}\right) / 0,5=b i \\
-c^{T}\left|x_{i}\right|^{T} \geq\left(a^{T} x_{i}^{T}-y_{i}\right) / 0,5=-b i \\
c_{j} \geq 0, \quad j=\overline{1,6}
\end{array}\right.
$$

Using Matlab 7.0.4 to solve problem (9), (10): $Z$ min $=2,457$.

Membership function of fuzzy moment of designing section. 
Table 2. Values of regression coefficients

\begin{tabular}{|c|c|c|c|c|c|c|}
\hline$\gamma_{j}$ & 1 & 2 & 3 & 4 & 5 & 6 \\
\hline$a_{j}$ & -0.1232 & 0.0179 & 0.0453 & 0.0035 & 0.0023 & 0.038 \\
\hline$c_{j}$ & 0.0027 & 0 & 0 & 0 & 0 & 0.0004 \\
\hline
\end{tabular}

From values of $\gamma_{j}\left(a_{j}, c_{j}\right)$ and mean value of $b, h, A_{S}, b^{2}, h^{2}, A_{S}^{2}$, we have the value of fuzzy moment $[\tilde{M}]$ :

$$
\begin{array}{r}
\tilde{R}=[\tilde{M}]=(-0.1232 ; 0.0027) \cdot b+(0.0179 ; 0) \cdot h+(0.0453 ; 0) . A_{S}+(0.0035 ; 0) \cdot b^{2}+ \\
+(0.0023 ; 0) \cdot h^{2}+(0.038 ; 0.0004) \cdot A_{S}^{2}=(6.061381 ; 0.090787) .
\end{array}
$$

where $a=6.061381$ is the central value of fuzzy moment $[\tilde{M}], c=0.090787$ is the amplitude of fuzzy moment $[\tilde{M}]$. Triangular-fuzzy number of moment $[\tilde{M}]$ is show in Fig. 6.

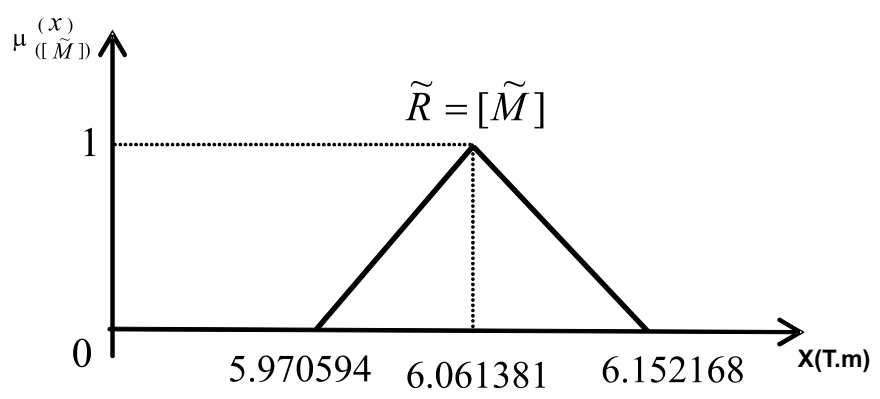

Fig. 6. Membership function of fuzzy moment of designing section

Based on values of $a$ and $c$ we have the membership function of fuzzy moment $[\tilde{M}]$ :

$$
\mu_{([\tilde{M}])}^{(x)}= \begin{cases}1-\frac{|x-6.061381|}{0.090787} & \text { when } 5.970594 \leq x \leq 6.152168 \\ 0 & \text { when } x \leq 5.970594 \text { and } x \geq 6.152168\end{cases}
$$

\subsection{Reliability assessment of beam at section $C$}

After determining two membership functions of two fuzzy sets $\tilde{M}_{c}$ and $[\tilde{M}]$, we evaluate the reliability of beam by new formula (Formula of area ratio), and compare with formulas in [2], [6], [7]. The Table 3 shows the results of the reliability of structure by different formulas.

Table 3. Results of formulas

\begin{tabular}{|c|c|c|c|c|c|c|c|}
\hline \multicolumn{2}{|c|}{ New Formula } & \multicolumn{2}{c|}{ Formula [2] } & \multicolumn{2}{c|}{ Formula [6] } & \multicolumn{2}{c|}{ Formula [7] } \\
\hline $\mathbf{P}_{\mathbf{S}}$ & $\mathbf{P}_{\mathbf{f}}$ & $\mathbf{P}_{\mathbf{S}}$ & $\mathbf{P}_{\mathbf{f}}$ & $\mathbf{P}_{\mathbf{S}}$ & $\mathbf{P}_{\mathbf{f}}$ & $\mathbf{P}_{\mathbf{S}}$ & $\mathbf{P}_{\mathbf{f}}$ \\
\hline 0,999621 & 0,000379 & 0,999621 & 0,000379 & 0,999611 & 0,000389 & 0,982513 & 0,017487 \\
\hline
\end{tabular}




\subsection{Comparison}

The numerical resuls calculating by new formula and by the formula in [2] are the same. The difference between new formula and the formula[6] is very small. In this example, the result calculated by [7] is less than $17 \%$ compared with the result obtained by the new formula.

\section{CONCLUSIONS}

Results obtained by the new formula and the formula [2] are the same. However, the new formula is applied easily in cases we can't define the intersectional part area of two sets $\tilde{S}$ and $\tilde{R}$, while the formula in [2] is not applicable.

It can be seen the "Formula of area ratio" is more general for evaluating safety of structures. Because this formula is established on the base of analysing not only the height ordinate $h$ but also calculates to the width base of intersectional part area, while the formula [7] only considers the height $h$. That is explained why the result obtained by the formula [7] less $17 \%$. The new formula uses fuzzy difference set $\tilde{M}=\tilde{R}-\tilde{S}$ its height is equal to unit, it mean normal fuzzy set, and so reflects exactly the essence of fuzzy numbers, while in the formula [6] height $\mathrm{h}$ is not equal to unit. So the formula proposed is believed for assessment of safety of structures.

\section{REFERENCES}

[1] Bernd Moller, Wolfgang Graf, Michael Beer, Safety Assessment of Structure in View of Fuzzy Randomness, Institute of Structural Analysis, Dresden University of Technology, Dresden Germany, (2003).

[2] Le Xuan Huynh, Fuzzy Set Theory application for Assessment of Safety of Structures, Proceedings of the Seventh National Congress on Mechanics, (2006) (in Vietnamese).

[3] Le Xuan Huynh, Application ability of Fuzzy Sets Theory for Quality Assessment of Construction Projects, Journal of Science Technology, National University of Civil Engineering Vietnam, 1 (2007) (in Vietnamese).

[4] Nguyen Dinh Xan, Reliability Determination of Structures Based on Fuzzy Set Theory, $P h D$ Thesis at NUCE, Hanoi, (2006) (in Vietnamese).

[5] Kwan - Ling - Lai, Fuzzy Based Structural Reliability Assessment, Structure Dept. China Engineering Consultants, Inc, Taipei, (1990).

[6] Nguyen Van Pho, A new method for determination of the reliability index of parameter distributed system, Vietnam Journal of Mechanics, 4 (2003).

[7] Weimin Dong, Wei-Ling Chiang, Haresh C. Snah, Assessment of Safety of Existing Buildings Using Fuzzy Set Theory, Dept. of Civil Engineering Stanford University, Stanford, CA, (1989).

[8] K. K. Yem, S. Ghoshray, G. Roig, A linear regression model using triangular fuzzy number coefficients, Department of Electrical \& Computer Engineering, Florida international university, Miami, USA, (1997). 